\section{Prevalencia de sensibilización alérgica a abeja, mosco y hormiga roja en niños con enfermedades alérgicas}

\section{RESUMEN}

Antecedentes: en población pediátrica, pocas veces se busca la sensibilización alérgica a insectos de los órdenes Hymenoptera y Culicidae.

Objetivo: establecer la prevalencia de sensibilización a abeja, mosco y hormiga roja en niños con enfermedades alérgicas.

Material y método: estudio retrolectivo y descriptivo, en el que se revisaron los registros de la reactividad cutánea a alergenos de abeja, mosco y hormiga en un servicio de Alergia Pediátrica de enero de 2009 a diciembre de 2011. Se estimó la frecuencia de sensibilización y sus respectivos intervalos de confianza de 95\% (IC95\%).

Resultados: se incluyeron 613 pacientes, $41 \%$ era del sexo femenino; la edad media del grupo fue de $6.2 \pm 3.7$ años. La sensibilización alérgica al mosco fue de 15\% (IC95\%: 12.4 a 18.1), a abeja de 14\% (IC95\%: 11.4 a 16.9 ) y a hormiga roja de $6 \%$ (IC95\%: 6.0 a 6.8); por sexo, la frecuencia no difirió significativamente. Los niños sensibles a las abejas o a las hormigas rojas tuvieron mayor edad que los no sensibilizados: 7.1 vs 6.0 años ( $p=0.011)$ y 7.4 vs 6.1 años $(p=0.042)$, respectivamente. Durante las estaciones de otoño e invierno predominó la sensibilización a la hormiga roja.

Conclusiones: hasta nuestro conocimiento, éste es el primer informe en México relacionado con la frecuencia de sensibilización a abejas, moscos y hormigas rojas realizado en niños. Además, nuestros resultados sugieren que se debe identificar de manera habitual la sensibilidad a estos insectos.

Palabras clave: abejas, Culicidae, himenópteros, hormigas, niños.

\author{
Leobardo Francisco Flores-Ruiz ${ }^{1}$ \\ Francisco Valdez-López ${ }^{2}$ \\ Martín Bedolla-Barajas ${ }^{3}$ \\ ${ }^{1}$ Servicio de Alergia e Inmunología Clínica. \\ ${ }^{2}$ Servicio de Pediatría. \\ Hospital Pediátrico de Sinaloa Dr. Rigoberto Aguilar \\ Pico, Culiacán, Sinaloa, México. \\ ${ }^{3}$ Servicio de Alergia e Inmunología Clínica, Hospital \\ Civil de Guadalajara Dr. Juan I Menchaca, Guadala- \\ jara, Jalisco, México.
}

Recibido: 28 de enero 2015

Aceptado: 14 de abril 2015

Correspondencia: Dr. Martín Bedolla Barajas Eulogio Parra 2330-301

44650 Guadalajara, Jalisco, México

drmbedbar@gmail.com

Este artículo debe citarse como

Flores-Ruiz LF, Valdez-López F, Bedolla-Barajas M. Prevalencia de sensibilización alérgica a abeja, mosco y hormiga roja en niños con enfermedades alérgicas. Revista Alergia México 2015;62:182-188. 
Objective: To determine the prevalence of sensitization to bees, mosquito and fire ants in children with allergic diseases.

Material and method: A retrolective and descriptive study was carried out in a pediatric allergy service. The registers of the cutaneous reactivity to bees, mosquitos and fire ants done among January 2009 to December 2011 were analyzed. The frequency of sensitization to insects and its confidence interval to $95 \%$ was estimated.

Results: The sample included 613 patients, $41 \%$ were girls. The age average of the group was $6.2 \pm 3.7$ years. The allergic sensitization to mosquito was $15 \%(\mathrm{Cl} 95 \%$ : $12.4-18.1)$, bees $14.2 \%$ (Cl 95\%: 11.4-16.9) and fire ants 6\% (Cl 95\%: 6.0-6.8) older that non-sensitized, 7.1 vs 6.0 years old $(p=0.011)$ and 7.4 vs 6.1 years old $(p=0.042)$, respectively. During fall and winter seasons, fire ant sensitization was observed with more frequency.

Conclusions: In our knowledge this is the first report in Mexico related with sensitization frequency to bees, mosquitos and fire ants carried out on children. Also, our results suggest that sensitization to these insects should be identified more often.

Key words: bee, Culicidae, Hymenoptera, ant, child.

\section{ANTECEDENTES}

Detectar a los alergenos que sensibilizan a los pacientes con enfermedades alérgicas ofrece la oportunidad de establecer medidas orientadas a evitar el contacto con esos agentes; pero también es una medición objetiva de las características del entorno con el que tiene contacto el paciente.

Phylum Arthropoda constituye $75 \%$ del reino animal, a él pertenecen los órdenes de los dípteros, constituido por moscas y mosquitos, y los himenópteros, conformado por abejas, hormigas, avispas y abejorros. ${ }^{1}$ El orden Diptera está constituido por aproximadamente 124,000 especies de moscas y mosquitos. ${ }^{2}$ En tanto, el orden himenóptero es uno de los más grandes órdenes de seres vivos, está conformado por cerca de 200,000 especies. ${ }^{3}$ En ambos casos, múltiples especies se han implicado con daños a la salud.
Diversos componentes químicos provenientes de insectos de los órdenes Hymenoptera y Culicidae se han implicado con reacciones alérgicas. En el caso de las abejas, su veneno está constituido principalmente por agua $(80 \text { a } 88 \%)^{4}$ y una diversidad de agentes químicos relacionados con sus efectos clínicos; entre ellos tenemos proteínas (fosfolipasa A2, hialurodinasa, fosfatasa ácida), péptidos (melitina, apamina) y aminas biógenas (histamina, adrenalina, noradrenalina, dopamina, serotonina, acetilcolina); 4,5 de ellos, los más abundantes son la melitina (Api $\mathrm{m}$ 4) y la fosfolipasa A2 (Api m 1), que representan entre 50 y $75 \%$ de la masa total del veneno de abeja. ${ }^{6}$ En cambio, los agentes tóxicos o alergénicos en las hormigas rojas son menos variados; en el género Formica se ha descrito la existencia de alcaloides de la piperidina (ácido fórmico) y algunas proteínas relacionadas con el veneno de avispas (fosfolipasa A1 y antígeno 5). ${ }^{1,5}$ Finalmente, en la saliva del mosquito se han encontrado más de 30 proteínas, muchas de ellas se consideran aler- 
génicas; estas proteínas incluyen antiagregantes plaquetarios (apirasa, Aed a 1), anticoagulantes (factor $\mathrm{Xa}$ ) y vasodilatadores (sialoquinina), entre otros. ${ }^{7,8}$ En consecuencia, la diversidad de alergenos mostrados por cada especie de insecto y la periodicidad de picaduras influyen de manera notoria en las cifras de sensibilización.

La prevalencia de picaduras de insectos en la población es muy elevada. Casi todas las personas, niños o adultos, han sufrido alguna vez una picadura por moscos; en menor cantidad, pero aún considerablemente, por abejas y en menos ocasiones por hormigas rojas. Son estos incidentes, las picaduras, los que hacen probable la sensibilización a algunos de sus productos biológicos.

En población pediátrica, la frecuencia de sensibilización a los moscos es de alrededor de 30\%; ${ }^{9-11}$ respecto de las abejas, la cantidad se estima en menos de $3 \%^{12-14}$ y en el caso de las hormigas rojas, en $5 \% .^{15}$

En México no existe evidencia suficiente que haya abordado el comportamiento epidemiológico de sensibilización alérgica a este tipo de insectos; asimismo, se sabe que la cantidad de alergólogos mexicanos que evalúan de manera habitual la sensibilización a moscos, abejas y hormigas es baja. ${ }^{16}$

El objetivo principal de este estudio es establecer las prevalencias de sensibilización alérgica a abeja, mosco y hormiga roja en niños con enfermedades alérgicas.

\section{MATERIAL Y MÉTODO}

Estudio retrolectivo y descriptivo, efectuado con datos correspondientes a niños y niñas menores de 18 años de edad, que padecían asma, rinitis alérgica, o ambas, residentes de la ciudad de Culiacán, Sinaloa. Todos los participantes fueron evaluados por un mismo alergólogo (FRL) durante el periodo comprendido de enero de 2009 a diciembre de 2011; sólo se incluyeron los niños que tuvieron al menos una prueba cutánea positiva a los alergenos probados.

El estudio se realizó en el Hospital Pediátrico de Sinaloa Dr. Rigoberto Aguilar Pico, situado en el noroeste de México en la ciudad de Culiacán, Sinaloa, que es una población ubicada a una altitud media sobre el nivel del mar de $64 \mathrm{~m}$. Los servicios del Hospital no sólo están dirigidos a los niños del estado de Sinaloa, sino también a los procedentes de Sonora, Baja California Sur, Nayarit y Durango; la mayoría de ellos procede de un medio socioeconómico bajo y no cuenta con seguridad social.

\section{Técnica de las pruebas cutáneas}

En la determinación de la sensibilización a abeja (Apis mellifera), mosco (Culex pipiens) y hormiga roja (Solenopsis invicta) se utilizó la técnica de punción. Los alergenos fueron de tipo glicerinado y en concentración 1:20 peso/volumen; histamina y solución de Evans sirvieron como control positivo y negativo, respectivamente.

Los alergenos habitualmente se sitúan sobre las regiones volares de los antebrazos y la punción se hizo con una lanceta calibrada marca AlkAbello $^{\circledR}$; la interpretación del resultado se hace a los 15 minutos posteriores a su aplicación. La prueba cutánea se considera positiva cuando el tamaño de la pápula originada en el sitio de aplicación de cada alergeno es mayor a $3 \mathrm{~mm}$ que el control negativo.

\section{Ética}

La investigación fue aprobada por el Comité de Ética e Investigación del Hospital Pediátrico de Sinaloa. 


\section{Análisis estadístico}

En las variables continuas se calculó la media y su desviación estándar. Para las variables nominales se estimó su proporción y sus intervalos de confianza de 95\% (IC95\%) y en su comparación se utilizó la prueba $\chi^{2}$ o la prueba exacta de Fisher, según fue necesario. En las variables continuas con distribución normal se utilizó la prueba t de Student. Los análisis se realizaron con el programa IBM SPSS, versión 20.0 para Windows (Armonk, NY, Estados Unidos).

\section{RESULTADOS}

Se incluyeron 613 pacientes; la edad media fue de $6.2 \pm 3.7$ años; 249/613 (41\%) eran niñas. La relación hombre:mujer fue 1.5 a 1 . Todos los niños estudiados tenían asma; la asociación asma y rinitis alérgica fue de $60 \%$.

La prevalencia de sensibilización a cualquier tipo de insecto fue de 184/613 (30\%); solamente a uno fueron $150 / 613(25 \%)$, a dos fueron $32 / 613(5 \%)$ y a los tres fueron $2 / 613(0.3 \%)$.

El insecto con mayor frecuencia de sensibilización fue el mosco: 94/613 (15\%; IC 95\%: 12.4 a 18.1), seguido por la abeja 87/613 (14\%; IC $95 \%: 11.4$ a 16.9$)$ y al final la hormiga roja 39/613 (6\%; IC 95\%: 6.0 a 6.8). De acuerdo con el sexo, no se observó diferencia estadísticamente significativa (Cuadro 1).

La media de edad de los niños sensibilizados a las abejas o las hormigas rojas fue significativamente mayor que la de los niños no sensibilizados (Cuadro 2). En relación con la sensibilización al mosco, no se observó diferencia.

El Cuadro 3 refleja que la estación del año se asoció significativamente con la sensibilización a la hormiga roja; con predominio durante el otoño y el invierno. La sensibilización a las abejas y a los moscos no mostró asociación.

\section{DISCUSIÓN}

Este estudio demuestra que la sensibilización alérgica a insectos como las abejas, los moscos o las hormigas en niños es un evento común.

Los estudios que han evaluado la frecuencia de sensibilización a insectos como abejas, hormigas y moscos son escasos y muestran resultados variables. Un estudio realizado en Irán, ${ }^{9}$ que incluyó una muestra de 31 niños y 55 adultos, ambos grupos con rinitis alérgica, mostró que la proporción de sensibilización a los moscos fue muy similar: 32 y $33 \%$, respectivamente. En la India $^{10}$ se estudiaron 918 pacientes con asma, rinitis alérgica o ambas, un poco menos de $25 \%$ fueron niños, el objetivo fue determinar los patrones de sensibilización a los aeroalergenos de la región; el mosco tuvo una frecuencia de 32\%.

Cuadro 1. Frecuencia de sensibilización alérgica a diferentes insectos de acuerdo con el sexo

\begin{tabular}{lccc}
\hline & \multicolumn{2}{c}{ Sexo } & \\
& Femenino & Masculino & Valor de $\mathbf{p}$ \\
$\mathbf{n = 2 4 9}(\mathbf{\%})$ & $\mathbf{n = 3 6 4}(\mathbf{\%})$ & \\
Abeja & $35(14.1)$ & $52(14.3)$ & 0.936 \\
Mosco & $37(14.9)$ & $57(15.7)$ & 0.787 \\
Hormiga roja & $12(4.8)$ & $27(7.4)$ & 0.196
\end{tabular}

Valor de $\mathrm{p}$ obtenido por la prueba $\chi^{2}$.

Cuadro 2. Asociación entre sensibilización a insectos y edad

\begin{tabular}{lccc}
\hline & \multicolumn{2}{c}{ Prueba cutánea } & \multirow{2}{*}{ Valor de p } \\
& Positiva & Negativa & \\
Abeja & $7.1 \pm 3.9$ & $6.0 \pm 3.6$ & 0.011 \\
Mosco & $6.8 \pm 4.0$ & $6.1 \pm 3.6$ & 0.083 \\
Hormiga roja & $7.4 \pm 3.8$ & $6.1 \pm 3.7$ & 0.042 \\
\hline
\end{tabular}

Los valores expresan media \pm desviación estándar en años. Valor de p obtenido por la prueba t de Student. 
Cuadro 3. Frecuencia de sensibilización a insectos de acuerdo con la estación del año

\begin{tabular}{lccccc}
\hline & \multicolumn{2}{c}{ Estación del año } \\
& $\begin{array}{c}\text { Primavera } \\
\mathbf{n = 1 4 7}(\mathbf{\%})\end{array}$ & $\begin{array}{c}\text { Verano } \\
\mathbf{n = 1 7 9}(\boldsymbol{\mathbf { 1 }})\end{array}$ & $\begin{array}{c}\text { Otoño } \\
\mathbf{n = 1 2 7}(\boldsymbol{\%})\end{array}$ & $\begin{array}{c}\text { Invierno } \\
\mathbf{n = 1 6 0}(\mathbf{\%})\end{array}$ & Valor de $\mathbf{p}$ \\
Abeja & $16(11)$ & $27(15)$ & $22(17)$ & $22(14)$ & 0.478 \\
Mosco & $24(16)$ & $27(15)$ & $19(15)$ & $24(15)$ & 0.986 \\
Hormiga roja & $1(0.7)$ & $8(4)$ & $11(9)$ & $19(12)$ & $<0.001$
\end{tabular}

Valor de $\mathrm{p}$ obtenido por la prueba $\chi^{2}$ o exacta de Fisher.

Un estudio de características similares al previo, pero realizado en Colombia, mostró que $27 \%$ de los pacientes estaban sensibilizados al mosco. ${ }^{11}$ Al contrastar los resultados obtenidos en este estudio con los de los estudios mencionados, la proporción de niños alérgicos al mosco es inferior, lo que probablemente se deba al tiempo y la frecuencia de exposición a la picaduras de estos insectos.

Respecto de las abejas, los estudios de prevalencia de sensibilización a himenópteros en niños también son escasos. En población no seleccionada, un estudio realizado con 1,175 niños italianos en edad escolar, a quienes se les practicaron pruebas cutáneas, demostró que $3 \%$ tuvo una prueba positiva contra el veneno de abeja. ${ }^{12}$ Este hallazgo contrasta notoriamente con nuestros resultados, pues la cantidad de niños alérgicos a las abejas fue de 14\%. Este comportamiento adelanta que la atopia en un factor de riesgo relevante en la sensibilización alérgica a abejas. En México, la frecuencia de sensibilidad a abejas se ha estudiado principalmente en población adulta. Por ejemplo, en un estudio analítico que comparó un grupo de apicultores con otro de no apicultores y utilizó diferentes concentraciones de veneno de abeja para realizar las pruebas cutáneas, la prevalencia de sensibilización fue de 16 vs $13 \%$ y 11 vs $7 \%$, según la concentración mayor y menor de alergeno utilizado. ${ }^{13}$ En otro estudio, la proporción de estudiantes de veterinaria sensibilizados a las abejas fue de $16 \% .{ }^{14}$ De acuerdo con estos datos y los nuestros, parece que para el caso de las abejas ni la actividad laboral, ni el tipo de alergeno utilizado o el área geográfica están relacionados con la frecuencia de sensibilización a las abejas.

La alergia a las hormigas rojas ha llamado de manera importante la atención en diversas regiones del mundo. En Estados Unidos, se analizaron los resultados de una muestra de pacientes con asma, rinitis alérgica o ambas, 38 adultos y 162 niños, y se encontró que $5 \%$ de ellos estaban sensibilizados a las hormigas. ${ }^{15}$ Este hallazgo es consistente con el nuestro, en el que $6 \%$ de los niños estaba sensibilizado. Asimismo, la población adulta mostró un comportamiento diferente; se ha visto que los estudiantes de veterinaria tienen una frecuencia mayor, que va de 25 a $31 \% .{ }^{11,14}$

La cantidad de alergólogos mexicanos que usan los alergenos de abeja, mosco y hormiga roja como escrutinio de sensibilización en los pacientes con enfermedades alérgicas es baja. Así lo demuestra un estudio de encuesta realizado con 53 médicos alergólogos distribuidos en México, donde poco más de $20 \%$ informó que incluían de manera rutinaria el alergeno de mosco, poco más de $15 \%$ el de abeja y aproximadamente $10 \%$ el de hormiga roja. ${ }^{16}$

Nuestros resultados permiten sugerir que en esta área geográfica o en otras con características similares a la nuestra estos agentes se integren 
a la batería de análisis de los niños con enfermedades alérgicas, porque su frecuencia de sensibilización fue comparable con la informada para alergenos procedentes de árboles, malezas, hongos, alimentos y algunos epitelios (en general inferior a 20\%), de acuerdo con un estudio realizado previamente en nuestra ciudad, ${ }^{17}$ en el que no se consideró a los alergenos de abeja, mosco y hormiga, lo que hubiera permitido verificar si nuestros resultados eran consistentes.

Al contrastar la prevalencia de sensibilización con el sexo de los pacientes, no encontramos diferencia estadísticamente significativa para cualquiera de los insectos analizados. Sin embargo, existe evidencia que señala que el riesgo de sensibilización está influido por el sexo masculino versus femenino, OR: 1.91 ; IC 95\%: 1.24 a 2.95. ${ }^{18}$ De manera más extensa, un metanálisis mostró que el sexo sí influye en la prevalencia de sensibilización; ésta fue mayor en los niños vs niñas (razón hombre-mujer, 1.39, IC 95\%: 1.13 a 1.71), y en los hombres frente a las mujeres (razón hombre-mujer, 1.40, IC 95\%: 1.22 a 1.61). ${ }^{19}$ Con excepción de la hormiga roja, en nuestro estudio sólo se encontró diferencia en la prevalencia de la enfermedad alérgica, porque ésta fue más común en los niños que en las niñas y no en la frecuencia de sensibilización a los alergenos.

Los niños con la prueba cutánea positiva al alergeno de abeja y hormiga roja tuvieron mayor edad que los niños con la prueba negativa. Esta conducta probablemente tiene que ver con el tiempo y la frecuencia de exposición a estos insectos; se ha visto que la edad guarda relación estrecha con la frecuencia de sensibilización atópica. ${ }^{10,18}$

La prevalencia de sensibilización a las abejas y los moscos no mostró predominio significativo por alguna estación del año en particular, pero en el caso de la hormiga roja, sí hubo preferen- cia significativa por la época invernal. Modelos similares al nuestro han mostrado que este comportamiento de variación estacional no es exclusivo de las hormigas. Por ejemplo, en un estudio donde se analizaron las historias clínicas de 1,891 pacientes se observó que los pacientes con alergia no respiratoria, la prevalencia de sensibilización alérgica fue significativamente menor durante el verano (23 vs 36\%, $\mathrm{p}=0.021$ ), y la prevalencia más alta tendía a ocurrir durante la primavera (40 vs 29\%, $\mathrm{p}=0.061$ ) en relación con el resto de las estaciones. ${ }^{20}$ Otro estudio evidenció que la reactividad cutánea y las concentraciones séricas de anticuerpos IgE específicos contra ácaros disminuyeron significativamente durante el mes de diciembre respecto a septiembre, $p<0.05$ y $p<0.05$, respectivamente. ${ }^{21}$

\section{Limitaciones}

Los porcentajes de sensibilización observados reflejan el grado de exposición previa a estos insectos. Sin embargo, debido a las características de este estudio, de ser retrolectivo, no pudo determinarse qué tan comúnmente los niños habían recibido picaduras previamente.

Una limitante adicional tiene que ver con las características de los alergenos utilizados en la detección de sensibilización, porque éstos pueden influir de manera considerable; en nuestro hospital se utilizan extractos provenientes del cuerpo entero de estos insectos que, al no ser estandarizados, carecen de la sensibilidad y la especificidad adecuadas.

Aunque la frecuencia de sensibilización a insectos fue considerable en los niños con enfermedades alérgicas, esta sensibilidad no refleja necesariamente que los síntomas alérgicos sean desencadenados por los alergenos provenientes de las partes de alguno insectos; en su lugar, sólo ponen de manifiesto la existencia de anticuerpos 
IgE específicos de estos insectos, que se desarrollan a través de las picaduras previas.

\section{CONCLUSIONES}

En nuestro conocimiento, éste es el primer informe en México relacionado con la frecuencia de sensibilización a insectos provenientes de los órdenes Hymenoptera y Culicidae realizado en niños. Nuestros resultados sugieren que se debe identificar de manera habitual la sensibilización a estos insectos.

\section{REFERENCIAS}

1. Monzón-Muñoz FJ, Blasco-Gil RM. Patología causada por artrópodos de interés toxinológico y alergológico. Bol SEA 1997;20:193-215.

2. Hernández-Ortiz V, Dzul-Cauich JF. Moscas (Insecta: Diptera). [Revista en la Internet] Consultado: 2015 Enero 5. Disponible en: www2.inecc.gob. $\mathrm{mx} /$ publicaciones/ libros/542/cap7.pdf

3. Orden: Hymenoptera. [En Internet] Consultado: 2015 Enero 5. Disponible en: http://www.taxateca.com/ordenhymenoptera.html

4. Peña L, Pineda M. E, Hernández M, Rodríguez-Acosta A. Toxinas naturales: abejas y sus venenos. AVFT [revista en Internet]. 2006 Jun [citado 2014 Dic 07]; 25(1):6-10. Disponible en: http://www.scielo.org.ve/scielo.php?script=sci_ arttext\&pid=S0798-02642006000100001\&lng=es.

5. Karabus S. Hypersensitivity to stinging insect. Curr Allergy Clin Immunol J 2012;25:132-138.

6. de Roodt AR, Salomón OD, Orduna TA, Robles Ortiz LE y col. Envenenamiento por picaduras de abeja. Gac Méd Méx 2005;141:215-222.

7. Crisp HC, Johnson KS. Mosquito allergy. Ann Allergy Asthma Immunol 2013;110:65-69.

8. Moncada-Álvarez LI, Salazar-Terreros MJ, López- Páez MC. Alergia en el humano inducida por la saliva de insectos de la familia Culicidae. Rev Fac Med 2011;59:133-148.

9. Bemanian $\mathrm{MH}$, Alizadeh-Korkinejad $\mathrm{N}$, Shirkhoda $\mathrm{S}, \mathrm{Na}-$ bavi $\mathrm{M}$, Pourpak Z. Assessment of sensitization to insect aeroallergens among patients with allergic rhinitis in Yazd City, Iran. Iran J Allergy Asthma Immunol 2012;11:253-258.

10. Kumar R, Sharan N, Kumar M, Bisht I, Gaur SN. Pattern of skin sensitivity to various aeroallergens in patients of bronchial asthma and/or allergic rhinitis in India. Indian J Allergy Asthma Immunol 2012;26:66-72.

11. Sánchez-Caraballo J, Diez-Zuluaga S, Cardona-Villa R. Sensibilización a aeroalergenos en pacientes alérgicos de Medellín, Colombia. Rev Alerg Mex 2012;59:139-147.

12. Novembre $E$, Cianferoni $A$, Bernardini R, Veltroni $M$, et al. Epidemiology of insect venom sensitivity in children and its correlation to clinical and atopic features. Clin Exp Allerg. 1998;28:834-838.

13. Becerril-Ángeles $M$, Núñez-Velázquez M, Marín-Martínez J, Grupo del Programa Nacional de Control de la Abeja Africanizada SAGARPA. Rev Alerg Mex 2013;60:164-167.

14. Arias-Cruz A, Monsiváis-Toscano G, Gallardo-Martínez G, González-Díaz SN, Galindo-Rodríguez G. Prevalencia de la alergia a la picadura de himenópteros en estudiantes de medicina veterinaria de la ciudad de Monterrey, Nuevo León, México. Rev Alerg Mex 2007;54:77-81.

15. Smith TS, Hogan MB, Welch JE, Corder WT, Wilson NW. Modern prevalence of insect sensitization in rural asthma and allergic rhinitis patients. Allergy Asthma Proc 2005;26:356-360.

16. Larenas-Linnemann $D$, Arias-Cruz A, Guidos-Fogelbach GA, Cid del Prado ML. Alérgenos usados en las pruebas cutáneas en México. Rev Alerg Mex 2009;56:41-47.

17. Zazueta-Aldapa ID, Espinoza-Escobar I. Sensibilización alergénica en niños con asma y rinitis alérgica, del Hospital General de Culiacán. Arch Salud Sin 2010;4:49-53.

18. Carvajal Urueña I, Díaz Vázquez C, Cano Garcinuño A, García Merino A, et al. Grupo de Estudio Aplicaciones de Phadiatop infant en la Alergia Infantil. Perfil de sensibilización alérgica en niños de 0 a 5 años con sibilancias o dermatitis atópica. An Pediatr (Barc) 2010;72:30-41.

19. Goldhahn K, Bockelbrink A, Nocon M, Almqvist C, et al. Sex-specific differences in allergic sensitization to house dust mites: a meta-analysis. Ann Allergy Asthma Immunol 2009;102:487-494.

20. Choi IS, Lee SS, Myeong E, Lee JW, et al. Seasonal variation in skin sensitivity to aeroallergens. Allergy Asthma Immunol Res 2013;5:301-308.

21. Nahm DH, Park HS, Kang SS, Hong CS. Seasonal variation of skin reactivity and specific IgE antibody to house dust mite. Ann Allergy Asthma Immunol 1997;78:589-593. 